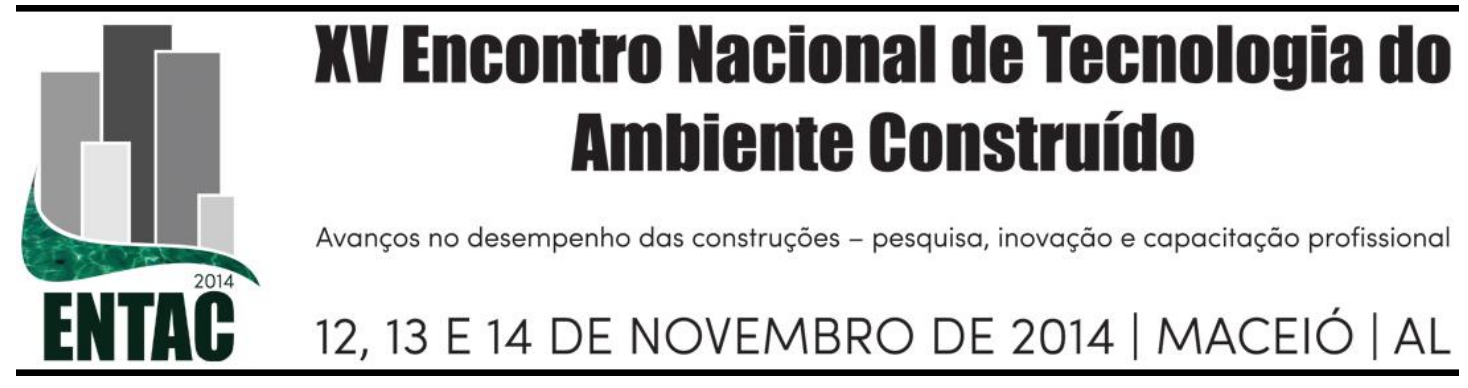

\title{
DESEMPENHO TERMO ENERGÉTICO DE PAREDES DE BLOCOS DE RESÍDUOS DE CONSTRUÇÃO E DEMOLIÇÃO
}

\author{
CALLEJAS, I. J. A. (1); DURANTE, L. C. (2); SILVA, R. A. F. (3); \\ OLIVEIRA, A. S. (4)
}

(1) UFMT, (65) 3615-8774, e-mail: ivancallejas@ig.com.br (2) UFMT, (65) 3615-8774, e-mail: luciane.durante@hotmail.com, (3) UFMT, (65) 3615-8774, e-mail: renataalves.au@gmail.com, (4) IFMT, (65) 3615-8774, e-mail: angela.oliveira@cba.ifmt.edu.br

\begin{abstract}
RESUMO
Com vistas à sustentabilidade ambiental, estudos tem procurado apresentar soluções técnicas que utilizem materiais provenientes de resíduos da construção e demolição (RCD). Nesse sentido, esta pesquisa teve por objetivo avaliar o desempenho termo energético de habitações de interesse social (HIS) composta por painéis de vedação executadas com blocos produzidos a partir de Resíduos de Construção e Demolição (RCD). Os específicos foram (a) caracterizar a transmitância e a condutividade térmica de parede composta por blocos de RCD, (b) avaliar os graus-hora de refrigeração e o conforto térmico de uma habitação de interesse social (HIS) com fechamentos em painéis de blocos de RCD com e sem revestimento, comparando-os com fechamento em painel convencional e (c) classificar a eficiência energética dessas habitações. A caracterização da transmitância e da condutividade térmica do painel de RCD e de blocos cerâmicos com revestimento seguiu o procedimento normativo com uso de câmara térmica. O desempenho termo energético foi avaliado por meio de simulações computacionais do projeto de HIS considerando painel de RCD, RCD com revestimento e convencional (bloco cerâmico). Os resultados indicaram que os graus-horas de refrigeração anual do Painel RCD foi 21,5\% superior ao painel convencional. O Painel RCD com revestimento reduziu a demanda anual para refrigeração de $20,60 \%$ em relação ao Painel RCD e de 3,5\% em relação ao Painel Convencional. A classificação da eficiência energética da HIS de Painel Convencional e de Painel RCD com revestimento foi Nível B e a do Painel RCD, Nível C. As três tipologias proporcionam $80 \%$ ou mais das horas em situação de desconforto térmico, evidenciando a necessidade de melhorias no projeto visando a obtenção de adequado conforto térmico, o que pode ser feito por meio da inserção de estratégias bioclimáticas, bem como a partir de melhorias no envelope da edificação, em especial, nas propriedades térmicas do bloco de RCD.
\end{abstract}

Palavras-chave: conforto térmico, materiais alternativos, simulação computacional, RCD.

\begin{abstract}
Aiming to improve environmental sustainability, studies have attempted to present technical solutions that use materials from construction and demolition $(C \& D)$ waste. In this sense, the objective of this research was to evaluate the thermal energetic performance of social housing composed of masonry fabricated with blocks produced from Recycled Construction and Demolition Waste (RCDW). Specific objectives were to (a) characterize the transmittance and thermal conductivity of wall composed of RCD blocks, (b) evaluate the degree- hour cooling and thermal comfort of a social housing (HIS) composed by panels with RCD blocks with and without external and internal mortar and compare them with conventional ceramic panel and (c) classifies the energy efficiency of those houses with those panels. The thermal transmittance and conductivity characterization of RCD RCD panel followed the normative procedure using thermal chamber. The thermo energetic performance was evaluated by means of computer project simulations considering social housing Panel with only RCD blocks, RCD blocks with external and internal mortar and conventional panels. The results indicated that the annual cooling degree-hours of RCD panel was $21.5 \%$ higher than the Conventional Panel. The RCD panel with internal and external
\end{abstract}


mortar reduced the annual cooling demand in $20.60 \%$ when compared to the Panel composed with only $R C D$ blocks and $3.5 \%$ compared to conventional panel. The social housing energy efficiency rating with Conventional Panel and RCD Panel with internal and external mortar was classified in Level B and Level $C$, with only RCD Panel. The annual thermal discomfort hours in the research buildings have achieved high levels of discomfort time (exceeding 80\%). It indicated the necessity to improve the building thermal comfort by introducing bioclimatic design strategies in the building project, as well as, improving in the building envelope, especially in the RCD block thermal properties.

Keywords: thermal comfort, alternative materials, thermo energetic computational simulation, $R C D$.

\section{INTRODUÇÃO}

Para projetar edificações energeticamente eficientes é necessário compreender os fenômenos de transferência de calor do ambiente externo para o interno, a fim de criar soluções que associem materiais e dimensões para adequação das envoltórias e, logo, redução do consumo energético e maior conforto térmico no interior das mesmas. Em termos normativos, a NBR 15220 - Parte 3 (ABNT, 2003) estabelece diretrizes construtivas para habitações de interesse social (HIS) aplicáveis na fase de projeto a partir da divisão do território brasileiro em oito Zonas Bioclimáticas (ZB), através de estratégias de condicionamento térmico passivo. $\mathrm{Na} Z \mathrm{ZB} 7$, região desta pesquisa, o desempenho térmico é um fator relevante, em virtude das altas temperaturas registradas durante todo o ano. No entanto, o que nela se observa é que os programas para HIS estão sendo implementados de forma padronizada, sem preocupação com o clima local e sem o atendimento das prescrições normativas (CALLEJAS et al., 2013).

Outro aspecto a ser considerado é que no Brasil o setor da construção civil é um grande gerador de resíduos, tanto no descarte de produtos fora de padrão pelas indústrias de materiais de construção, quanto nos reparos e demolições realizadas nos canteiros de obras. Destaca-se que apenas um terço deste material é destinado para os aterros públicos, sendo o restante geralmente depositado em áreas ilegais (LEITE et al., 2011). Uma alternativa para amenizar esse impacto ambiental é a reciclagem dos resíduos, utilizando-os como agregado reciclado (SANTOS et al., 2013). Segundo a NBR 15.116 (ABNT, 2004), agregado reciclado é um material granular proveniente do beneficiamento de resíduos de construção ou demolição de obras civis, que apresenta características técnicas para a aplicação em obras de edificação.

Uma importante ação com vistas à sustentabilidade, em especial a urbana, é a utilização dos materiais provenientes da construção e demolição como agregado na produção de blocos ou tijolos. Os reciclados da construção e demolição (RDC), como são chamados, podem apresentar inúmeras vantagens, tais como menor consumo de cimento em seu processo de fabricação, ausência do processo de queima, pois o bloco precisa ser somente curado, características físicas e mecânicas compatíveis com as dos blocos tradicionalmente produzidos, dentre outras (SANTOS et al., 2013). Algumas pesquisas têm destacado que a substituição dos agregados naturais graudos e miudos por agregados reciclados em níveis de $25 \%$ e $50 \%$ tem pouco efeito sobre a resistência à compressão, embora níveis mais elevados a reduzam substancialmente (SOUTSOS et al.,2011; LEIVA et al., 2013). Assim, o agregado do RCD é um material alternativo e atraente para ser utilizado em novos edifícios, especialmente nos fechamentos verticais.

É possível encontrar uma série de pesquisas dedicadas a caracterizar física e mecânicamente estes materiais (POON et al., 2002; LEIVA, et al., 2012). Em estudo recente, Santos et al. (2012) avaliou a viabilidade de aproveitamento dos RCD como agregado na fabricação de tijolos de vedação em Cuiabá/MT. Por outro lado, nota-se que pouco esforço tem sido feito para caracterizar as propriedades térmicas de blocos de 
$\mathrm{RCD}$, especialmente a sua transmitância térmica e condutividade térmica, bem como o comportamento térmico da edificação com a utilização de blocos de RCD.

Diante deste cenário, esta pesquisa teve por objetivo geral avaliar comparativamente o desempenho termo energético de habitação de interesse social (HIS) com fechamentos verticais de blocos produzidos a partir de Resíduos de Construção e Demolição (RCD) e de fechamento convencional de blocos cerâmicos revestidos interna e externamente. Os objetivos específicos foram (a) caracterizar a transmitância térmica e condutividade térmica de paredes de blocos de RCD com e sem revestimento e de blocos cerâmicos revestidos interna e externamente, (b) avaliar os graus-hora de refrigeração e o conforto térmico da HIS com fechamentos em painéis de RCD com e sem revestimento, comparando-os com fechamento em painel convencional de blocos cerâmicos revestidos interna e externamente e (c) classificar a eficiência energética dessas habitações segundo os critérios do Regulamento Técnico da Qualidade - RTQ-R.

\section{MATERIAIS E MÉTODOS}

\subsection{Resíduo de Construção e Demolição (RCD) e traço do concreto do bloco}

De acordo com Santos et al. (2013), a confecção dos agregados de RCD foi realizada selecionando os resíduos de construção para o processo de britagem. Foram escolhidos resíduos de argamassa, concreto e tijolo cerâmico (Figuras 1 e 2). Após o beneficiamento, o material britado foi dividido em três grupos granulométricos passantes nas peneiras $\# 0,15$ e $0,075 \mathrm{~mm}$ (pó), nas peneiras $\# 0,6$ e 0,3 $\mathrm{mm}$ (areia) e na peneira \#4,8 e 2,4-1,2 mm (pedrisco). Os ensaios físicos indicaram a seguinte caracterização do agregado de RCD: absorção de 12,26\%, massa unitária compactada de $1,48 \mathrm{~g} / \mathrm{cm}_{3}$, diâmetro máximo característico de $4,8 \mathrm{~mm}$ e módulo de finura de 3,04.

Para a definição do traço ideal, Santos et al. (2013) realizou um empacotamento experimental adaptado por Costa (2006), misturando três grupos granulométricos em diferentes porcentagens, até a determinação da combinação com maior massa unitária e consequentemente, menor índice de vazios. A combinação granulométrica dos materiais escolhidos foi $15 \%$ de pó, $47 \%$ de areia e $38 \%$ de pedrisco, cujo índice de vazios e massa específica foram $41,48 \%$ e $2,50 \mathrm{~g} / \mathrm{cm}^{3}$, respectivamente. O traço escolhido foi 1:9 (1:1,35:4,23:3,42; cimento:pó:areia:pedrisco) em massa, com fato água/cimento 1,32.

\subsection{Confecção dos blocos}

O concreto foi fabricado manualmente e moldado em uma prensa hidráulica para confecção dos blocos (Figuras 3 e 4). Os testes de resistência à compressão foram realizados de acordo com a NBR 6136 (2014) e foram superiores a 4MPa, classificandoos como Classe B, para uso estrutural em parede acima do nível do solo. Os testes físicos indicaram que a massa específica saturada/ seca, relação de vazios e de absorção de água por imersão foram de $1,6 \mathrm{~g} / \mathrm{cm}^{3}, 1,48 \mathrm{~g} / \mathrm{cm}^{3}, 26,75 \%$ e $12,5 \%$, respectivamente.

\section{Figura 1 - Processo Figura 2 - Mistura de britagem RCD agregada de RCD}
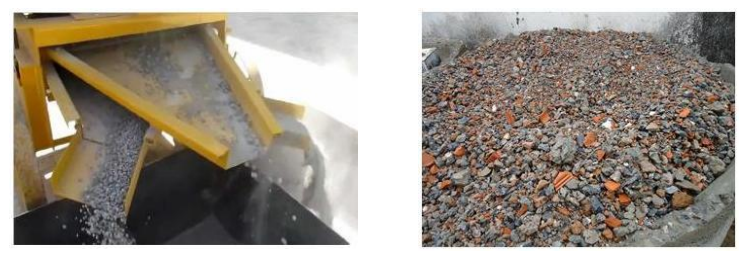

Figura 3 - Bloco de RCD na prensagem

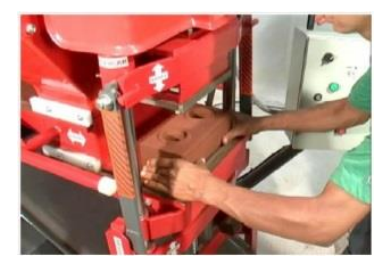

Figura 4 - RCD $25 \times 12,5 \times 7,5 \mathrm{~mm}$

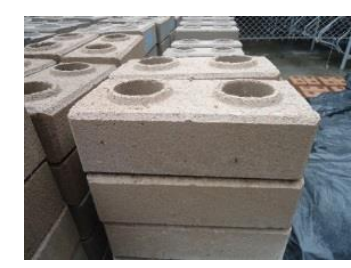

Fonte: Santos et al. (2013) 


\subsection{Determinação das propriedades térmicas por meio de câmara térmica}

Construiu-se uma câmara térmica teste para determinação da transmitância e condutividade térmica (Figuras 5 e 6), adaptada de Rupp (2009), com dimensões internas de $65 \times 50 \times 50 \mathrm{~cm}$, em placas de MDF $16 \mathrm{~mm}$, revestidas internamente com poliestireno expandido $(25 \mathrm{e} 50 \mathrm{~mm})$ envolvido em folhas de alumínio para proporcionar isolamento térmico do meio exterior. Uma lâmpada de infravermelho (250W) é a fonte de calor no interior da câmara e um dimmer ajusta a intensidade da luz de infravermelho. Um dos lados da câmara é fechado com o material a ser ensaiado, que fica exposto à radiação infravermelha emitida pela lâmpada durante o ensaio. A câmara térmica é envolvida por outra câmara, denominada "câmara ambiental", com dimensões

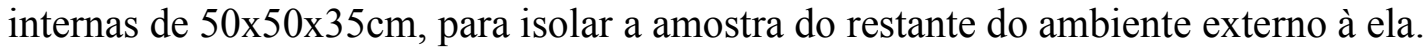

Foram confeccionadas amostras do fechamento vertical de blocos de RCD de 40x40cm, devidamente posicionadas na câmara acima descrita, para realização do ensaio. No total foram ensaiadas três amostras, sendo selecionada para apresentação neste artigo, aquela cujos resultados foram os mais desfavoráveis, ou seja, aquela em que se obtiveram os maiores valores de transmitância térmica. Também foi ensaiada uma amostra de painel com blocos cerâmicos de 8 furos $9 \times 19 \times 19 \mathrm{~cm}$, de meia vez, revestida interna e externamente com argamassa de $2,5 \mathrm{~cm}$ para confirmação dos valores normativos (teórico) e que permitem a comparação direta entre as amostras.

Os ensaios de transmitância térmica foram realizados utilizando-se a técnica de medição de fluxo de calor de acordo com a ISO 9869 (ISO, 1994). As medições foram realizadas durante 72 horas (três dias), a cada 5 minutos visto que este intervalo foi considerado adequado para captar variação dos parâmetros ambientais pesquisados.

Realizaram-se ensaios em duas posições da amostra de RCD: na seção sólida do bloco (sem a presença de vazios internos no painel) - RCD 1, e na seção vazada (câmara de ar) - RCD 2. Na amostra de bloco cerâmico, o ensaio foi realizado no centro da amostra (seção sólida e câmara de ar).

Foram instalados dois sensores de temperatura superficial (TMC-20-HD, OnsetComp) na posição central da amostra e voltados para o interior da câmara (centrados com a fonte de calor) para medir a temperatura superfical interna $\left(\mathrm{T}_{\mathrm{si}}\right)$, adequadamente protegidos da exposição direta à energia térmica da fonte de calor (Figura 7). Outros dois sensores foram fixados na face exterior da amostra, voltados para a câmara ambiental para medir a temperatura superficial externa $\left(\mathrm{T}_{\mathrm{se}}\right)$. Um sensor de fluxo de calor (q) (Hukseflux) foi fixado entre os dois sensores de temperatura superficial (Figura 8). Os sensores de temperatura superficial foram conectados a registradores automáticos (U12-13, Onsetcomp) e o sensor de fluxo de calor foi conectado ao registrador automático U30 (Onsetcomp), por meio de um amplificador de tensão.
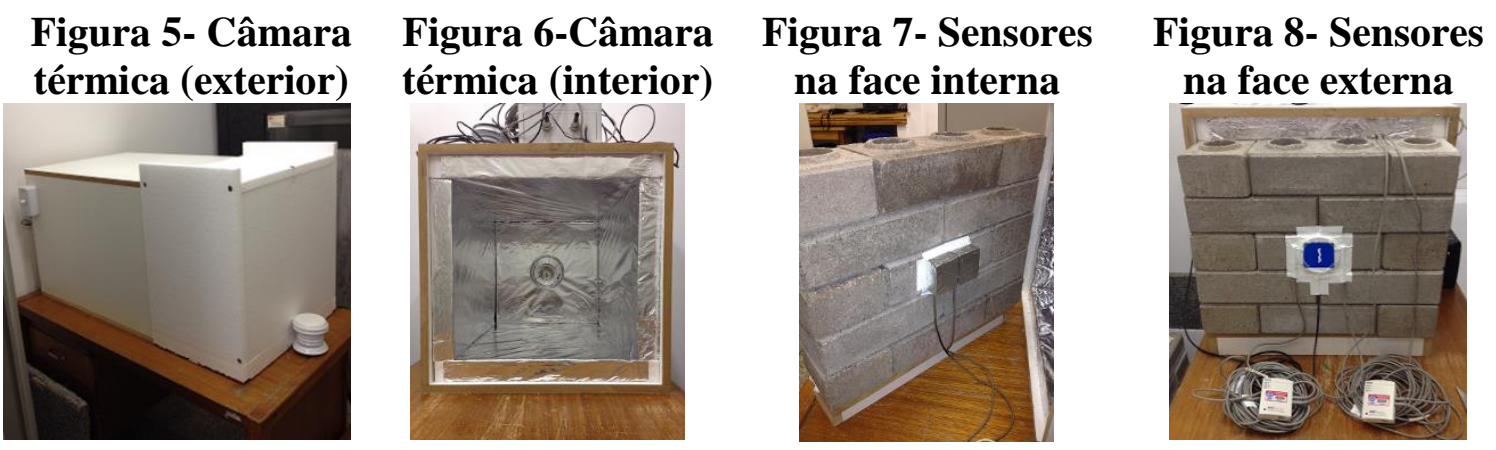

Fonte: do autor. 
A determinação da transmitância térmica $\left(U_{n}\right)$ foi feita através do método da média progressiva de acordo com a ISO 9869 (ISO, 1994), por meio da Equação 1, onde $T_{s i}$ é a temperatura média superficial interna, $T_{s e}$ é a temperatura média superficial externa, $q$ é o fluxo de calor entre os dois lados do elemento testado e $j$ representa cada uma das medições realizadas durante o teste de elemento (painél de vedação).

$$
U_{n}=\frac{\sum_{j=1}^{n} q_{j}}{\sum_{j=1}^{n}\left(T_{s i j}-T_{s e j}\right)}
$$

Como o valor de $\mathrm{U}$ a ser calculado converge assintoticamente para o valor final de $U_{n}$ e como se está interessado apenas no valor médio final, utilizou-se o conceito de incerteza combinada para estimar o erro introduzido na medição devido ao erro dos sensores, do desvio padrão observado em decorrência da variação ambiental durante as últimas 24 horas do experimento. A norma NBR ISO 17025 (ABNT, 2005) prescreve que a incerteza da medição pode ser feita assumindo-se o erro de calibração de instrumentos declarado pelos fabricantes, mais o desvio padrão (s.d) do período médio considerado de $U_{n}$. A incerteza estatística de medição (incerteza expandida) pode ser estimada assumindo um fator de abrangência (k) igual a 2, sendo este um valor típico neste tipo de medição. $O$ uso deste fator de abrangência leva a um nível de confiança de $95 \%$, aproximadamente. $\mathrm{O}$ valor de $\mathrm{U}$ final pode ser expresso pela Equação 2, em que $\delta U_{n}$ representa a incerteza total da estimativa de $U_{n}$, denominada de incerteza combinada (BAKER, 2011).

$$
\mathrm{U}=\mathrm{U}_{\mathrm{n}} \pm \mathrm{k} . \delta \mathrm{U}_{\mathrm{n}}
$$

\subsection{Desempenho termoenergético}

Os blocos de RCD caracterizados termicamente nesta pesquisa e propostos por Santos et al. (2003) tem sua produção destinada para a implantação de novas unidades habitacionais na Vila Tecnológica do Instituto Federal de Mato Grosso - Campus São Vicente - MT/ Brasil, com área de 37,56 m², distribuída em sala, cozinha, banheiro e dois quartos.

O projeto da HIS (Figura 9) teve seu desempenho termoenergético avaliado por meio de simulação computacional utilizando-se o programa EnergyPlus. Para a modelagem da edificação utilizou-se o Plug-in OpenStudio, que permite a edição e visualização em maquete eletrônica no SketchUp 8 (Figura 10).

Figura 9 - Planta baixa da HIS

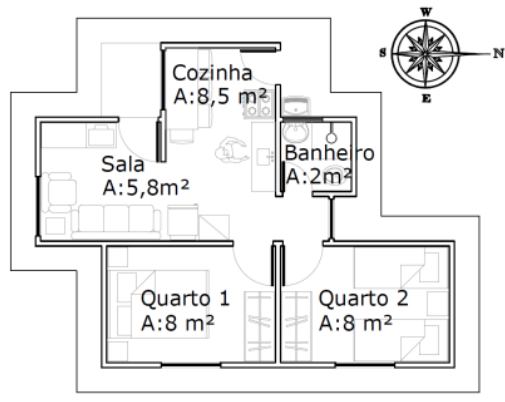

Fonte: do autor.

\section{Figura 10 - Maquete eletrônica da HIS}

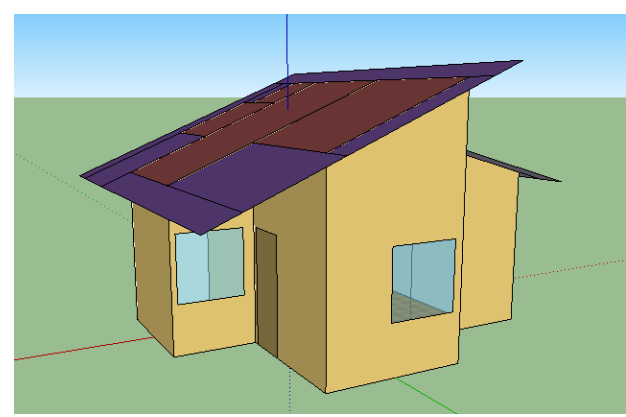

Fonte: do autor.

Para avaliar o desempenho térmico da edificação, utilizou-se a variável ambiental denominada de temperatura operativa $\left(\mathrm{T}_{0}\right)$ que é a temperatura teórica obtida pela média aritmética das temperaturas do ar e radiante do interior dos ambientes. A temperatura 
operativa foi avaliada para os ambientes de permanência prologada (sala, cozinha e quartos), conforme recomendação do RTQ-R (2010) que serviu de referência a para o cálculo dos graus-horas de desconforto e de resfriamento dos ambientes da edificação. Segundo BUYUKALACA et al. (2001), graus-hora é um parâmetro climático de desempenho definido como o somatório das diferenças de temperatura, quando esta se encontra abaixo ou acima de uma temperatura base $\left(\mathrm{T}_{\mathrm{b}}\right)$, calculado conforme Equação 3 , em que $\mathrm{T}_{0}$ é a temperatura operativa média horária e $\mathrm{T}_{\mathrm{b}}$ a temperatura base. A partir deste parâmetro é possível calcular a porcentagem de horas de desconforto, bem como as horas necessárias para refrigeração da edificação.

$$
\mathrm{GH}=\Sigma\left(\mathrm{T}_{\mathrm{o}}-\mathrm{T}_{\mathrm{b}}\right)
$$

Para o computo das horas de desconforto por calor, partiu-se dos valores proposto por GIVONI (1992) para países com clima quente e em desenvolvimento, que sugere para o verão, em situação de umidade alta, valores limites superior de temperatura de conforto entre 25 e $26^{\circ} \mathrm{C}$, sendo o útlimo adotado como referência, por ser a temperatura base para cálculo dos graus-hora de refrigeração do ambiente, conforme indicado pelo RTQR (2010) e, também, pelo fato de que as pessoas estão aclimatadas ao clima local.

Foram realizadas simulações computacionais do projeto da HIS com três tipologias de fechamentos verticais: a) "painel convencional" (blocos cerâmicos de 8 furos 9x19x19cm, de meia vez, revestidos interna e externamente com argamassa de 2,5cm, conforme usualmente seria construída na região), b) "painel RCD" (blocos de RCD sem revestimento) e c) "painel RCD+revestimento" (aos quais foram acrescidos o revestimento interno e externo de $2,5 \mathrm{~cm})$. Os demais parâmetros construtivos mantiveram-se inalterados.

\section{RESULTADOS E DISCUSSÃO}

\subsection{Transmitância e condutividade térmica dos Blocos de RCD}

Os resultados do ensaio na seção do painel composta pela parte sólida do bloco (RCD 1) indicam que os valores calculados convergem assintoticamente para o valor final (Figura 11a). A diferença média de temperatura entre as faces foi de $\sim 13,5^{\circ} \mathrm{C}$, o fluxo de calor médio foi de $76 \mathrm{~W} / \mathrm{m}^{2}$, o valor médio de $U_{n}$ e a incerteza estatística da medição do ensaio foram de $5,69 \mathrm{~W} / \mathrm{m}^{2} \mathrm{~K} \pm 0,70 \mathrm{~W} / \mathrm{m}^{2} \mathrm{~K}$ (Quadro 1).

Nos resultados do ensaio com os sensores posicionados na seção do bloco com câmara de ar (RCD 2), o comportamento foi semelhante ao realizado na região sólida (Figura $11 \mathrm{~b}$ ), com temperaturas superficiais interna e externa semelhantes. Contudo, o resultado da transmitância térmica foi menor, como esperado, visto que a propagação térmica é maior em meios sólidos (Quadro 1). O valor médio de $U_{n}$ e a incerteza estatística da medição do segundo ensaio foram de $5,23 \mathrm{~W} / \mathrm{m}^{2} \mathrm{~K} \pm 0,60 \mathrm{~W} / \mathrm{m}^{2} \mathrm{~K}$.

No ensaio realizado no bloco cerâmico, as temperaturas superficiais interna e externa foram superior ao bloco de RCD, com diferença média de temperatura entre as faces de $\sim 16,4^{\circ} \mathrm{C}$ e fluxo de calor médio de $81 \mathrm{~W} / \mathrm{m}^{2}$ (Figura $11 \mathrm{c}$ ) $\mathrm{O}$ valor médio de $\mathrm{U}_{\mathrm{n}}$ e a incerteza estatística da medição do ensaio foram de $4,92 \mathrm{~W} / \mathrm{m}^{2} \mathrm{~K} \pm 0,56 \mathrm{~W} / \mathrm{m}^{2} \mathrm{~K}$ (Quardo 1), inferior aos resultados encontrados na região sólida e câmara de ar do RCD.

A transmitância térmica global do bloco (U) foi calculada ponderando-se a transmitância obtida nos testes RCD 1 e RCD 2 pelas respectivas áreas de secção transversal do bloco. $\mathrm{O}$ valor de $\mathrm{U}_{\mathrm{T}}$ encontrado foi de $5,48 \mathrm{~W} / \mathrm{m}^{2} \mathrm{~K} \pm 0,32 \mathrm{~W} / \mathrm{m}^{2} \mathrm{~K}$.

A transmitância térmica total $\left(\mathrm{U}_{\mathrm{T}}\right)$ do painel $\mathrm{RCD}$ e bloco cerâmico foi de $2,89 \mathrm{~W} / \mathrm{m}^{2} \mathrm{~K} \pm 0,32 \mathrm{~W} / \mathrm{m}^{2} \mathrm{~K}$ e $2,40 \mathrm{~W} / \mathrm{m}^{2} \mathrm{~K} \pm 0,56 \mathrm{~W} / \mathrm{m}^{2} \mathrm{~K}$, respectivamente. $\mathrm{O}$ valor foi 
obtido somando-se a transmitância térmica externa e interna do ar. De acordo com a NBR 15220-3 (ABNT, 2003), o valor teórico da transmitância térmica total do bloco cerâmico com revestimento em ambas as faces com $2,5 \mathrm{~cm}$ é de $2,49 \mathrm{~W} / \mathrm{m}^{2} \mathrm{~K}$, valor próximo ao determinado no ensaio e dentro do intervalo de confiança.

A transmitância térmica total do painel RCD foi superior ao da parede composta por blocos cerâmicos de 8 furos revestidos com argamassa interna e externamente, com espessura total variando de 0,14 a $0,15 \mathrm{~m}$ e transmitância térmica total entre 2,24 a $2,49 \mathrm{~W} / \mathrm{m}^{2} \mathrm{~K}$, cujo valor normativo foi confirmado no ensaio (Figura 11c).

\section{Quadro 1 - Valores médios das últimas 24h na seção sólida do bloco de RCD 1/ com câmara de ar do bloco RCD 2 e na seção central do bloco cerâmico}

\begin{tabular}{|c|c|c|c|}
\hline Variável/ propriedade térmica & $\begin{array}{c}\text { RCD 1: } \\
\text { sensores na } \\
\text { seção sólida } \\
\end{array}$ & $\begin{array}{l}\text { RCD 2: sensores } \\
\text { na seção com } \\
\text { câmara de ar } \\
\end{array}$ & $\begin{array}{l}\text { Bloco cerâmico: } \\
\text { sensores na } \\
\text { seção central } \\
\end{array}$ \\
\hline Temperatura interna & $58,74^{\circ} \mathrm{C}$ & $55,33^{\circ} \mathrm{C}$ & $60,71^{\circ} \mathrm{C}$ \\
\hline Temperatura externa & $42,47^{\circ} \mathrm{C}$ & $42,53^{\circ} \mathrm{C}$ & $44,31^{\circ} \mathrm{C}$ \\
\hline Fluxo de calor & $76,35 \mathrm{~W} / \mathrm{m}^{2}$ & $66,93 \mathrm{~W} / \mathrm{m}^{2}$ & $80,71 \mathrm{~W} / \mathrm{m}^{2}$ \\
\hline Valor de U médio $\left(\mathrm{U}_{\mathrm{n}}\right)$ & $5,69 \mathrm{~W} / \mathrm{m}^{2} \mathrm{~K}$ & $5,23 \mathrm{~W} / \mathrm{m}^{2} \mathrm{~K}$ & $4,92 \mathrm{~W} / \mathrm{m}^{2} \mathrm{~K}$ \\
\hline Incerteza $\delta U_{n}$ & $0,35 \mathrm{~W} / \mathrm{m}^{2} \mathrm{~K}$ & $0,30 \mathrm{~W} / \mathrm{m}^{2} \mathrm{~K}$ & $0,28 \mathrm{~W} / \mathrm{m}^{2} \mathrm{~K}$ \\
\hline \begin{tabular}{l|l}
$\mathrm{U}_{\text {err__ }} \mathrm{T}_{\text {si }}\left(\right.$ erro do sensor $\left.0,25^{\circ} \mathrm{C}\right)$ \\
\end{tabular} & 5,80 & 5,33 & 5,03 \\
\hline g $\mathrm{U}_{\text {err_- }} \mathrm{T}_{\mathrm{se}}$ (erro do sensor $0,25^{\circ} \mathrm{C}$ ) & 5,80 & 5,33 & 5,00 \\
\hline : $\mathrm{U}_{\text {err_Q }} \mathrm{Q}$ (erro do sensor 5\%) & 5,98 & 5,49 & 4,67 \\
\hline 鳬 Desvio padrão (s.d.) & 0,13 & 0,06 & 0,04 \\
\hline Erro percentual $\left(100 . \delta \mathrm{U}_{\mathrm{n}} / \mathrm{U}_{\mathrm{n}}\right)$ & $6,50 \%$ & $5,75 \%$ & $5,73 \%$ \\
\hline
\end{tabular}

Figura 11 - (a) Ensaio na seção sólida do bloco RCD 1, (b) na seção com câmara de ar do bloco RCD 2 e (c) na parede de bloco cerâmico com revestimento

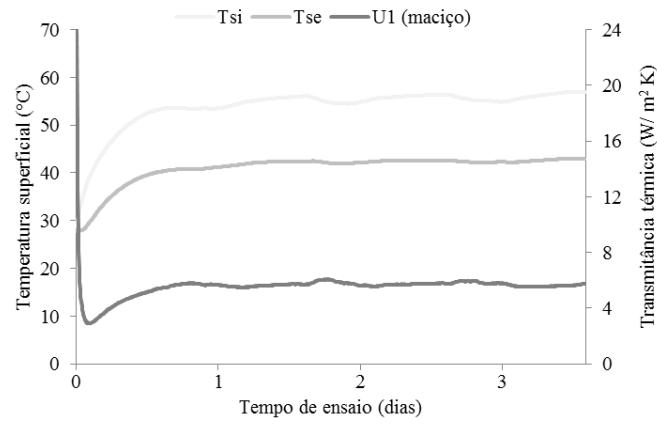

(a)

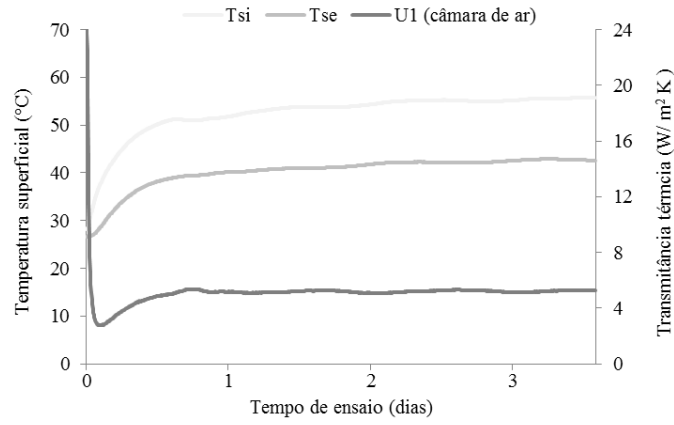

(b)

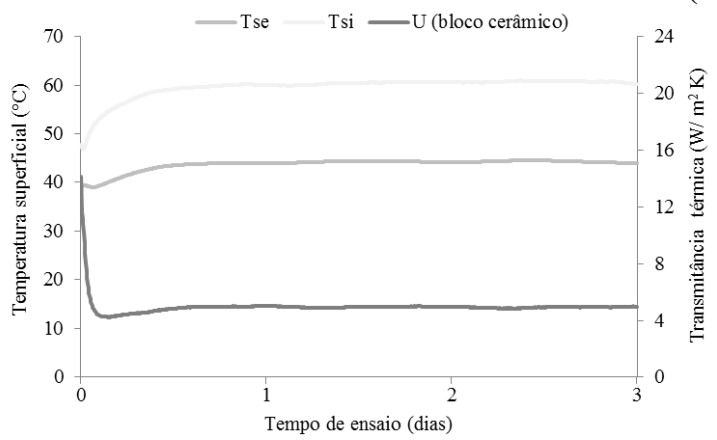

(c) 
Na zona bioclimática 7, região de implantação da HIS, a NBR 15575-3 (ABNT, 2003) estabelece que se o índice de absortância $(\alpha)$ inferior a 0,6 , a transmitância total deve ser inferior a $3,7 \mathrm{~W} / \mathrm{m}^{2} \mathrm{~K}$. Caso o índice de absortância exceda 0,6 , a transmitância total reduz-se para $2,5 \mathrm{~W} / \mathrm{m}^{2} \mathrm{~K}$, com vistas a evitar o ganho excessivo de calor no interior das habitações. Como a parede de RCD tem coloração cinza escuro $(\alpha>0,6)$ e o resultado do ensaio indicou transmitância total da parede RCD superior a $2,5 \mathrm{~W} / \mathrm{m}^{2} \mathrm{~K}$, o painel RCD não atende aos parâmetros indicados na referida norma.

No painel RCD+revestimento foi introduzido o revestimento interno e externo de $2,5 \mathrm{~cm}$ de espessura, obtendo-se transmitância térmica da parede seja de aproximadamente de $2,5 \mathrm{~W} / \mathrm{m}^{2} \mathrm{~K}$, valor este que atende a norma e se aproxima da transmitância das paredes compostas por blocos cerâmicos e argamassa.

A condutividade térmica do bloco foi determinada multiplicando-se o valor de $U_{n}$ pela espessura do bloco, o que resultou no valor de $\lambda$ igual a $0,68 \mathrm{~W} / \mathrm{mK} \pm 0,04 \mathrm{~W} / \mathrm{mK}$. Como esperado, o bloco de RCD apresentou menor valor de condutividade térmica do que o bloco de concreto convencional, estabelecido em $\lambda_{\mathrm{c}}=1,75 \mathrm{~W} / \mathrm{mK}$ pela NBR $15220-2$ (ABNT, 2003). Isso ocorre devido à presença de agregados com menor densidade e condutividade térmica, como cerâmica e gesso que compõem o RCD e, também, devido à maior porosidade da mistura.

\subsection{Simulação do desempenho termo energético}

A análise do parâmetro graus-horas de refrigeração (Figura 12) indicou que as três tipologias de fechamentos verticais estudadas demandaram maior quantidade de horas para refrigeração no mês de novembro e menor no mês de julho, comportamento que está diretamente relacionado com o clima da região (Tropical Continental).

Constatou-se que em todos os meses do ano, a HIS com painel RCD apresentou maior demanda de refrigeração que a HIS com painel convencional. Com a introdução da argamassa interna e externa ao bloco, no painel RCD+revestimento, observou-se que o desempenho foi melhorado, fazendo com que a demanda de refrigeração fosse inferior ao do edificício com painel convencional. Destaca-se que as argamassas de revestimento são idênticas: cimento portland e areia lavada.

Em termos anuais, a HIS composta por painel convencional demandou $17.174^{\circ} \mathrm{Ch}$ de refrigeração. A HIS composta por painel RCD apresentou demanda anual 21,50\% superior à de painel convencional $\left(20.870^{\circ} \mathrm{Ch}\right)$. A introdução de revestimento interno e externo reduziu a demanda anual para refrigeração em $20,60 \%$ em relação ao painel $\mathrm{RCD}$ e $3,5 \%$ em relação ao painel convencional $\left(16.567^{\circ} \mathrm{Ch}\right)$.

Aplicando-se o RTQ-R, a envoltória da edificação com painel convencional classificase com o Nível B de eficiência. Já a edificação com painel de blocos de RCD sem revestimento externo e interno classifica-se com Nível C, acarretando redução no nível de desempenho em relação ao painel convencional. A edificação com painéis de RCD e revestimento interno e externo, além de apresentar redução nos graus-horas de refrigeração, classifica-se como Nível B, mantendo o mesmo nível da HIS com paineis convencionais.

A análise do parâmetro de horas de desconforto (Figura 13) indica que nas edificações simuladas, em todos os meses do ano, $80 \%$ das horas estiveram dentro da faixa de desconforto. Com exceção do mês de julho, nos demais meses do ano, as porcentagens de horas de desconforto proporcionadas pelas HIS são inadequadas, destacando-se que nos mêses de outubro a janeiro, os níveis foram superiores a $90 \%$. Constata-se, então, que a busca por melhores níveis de desempenho termoenergético não garantem necessariamente níveis adequados de conforto térmico no interior da edificação. 
Figura 12 - Graus-horas de refrigeração anual dos paineis

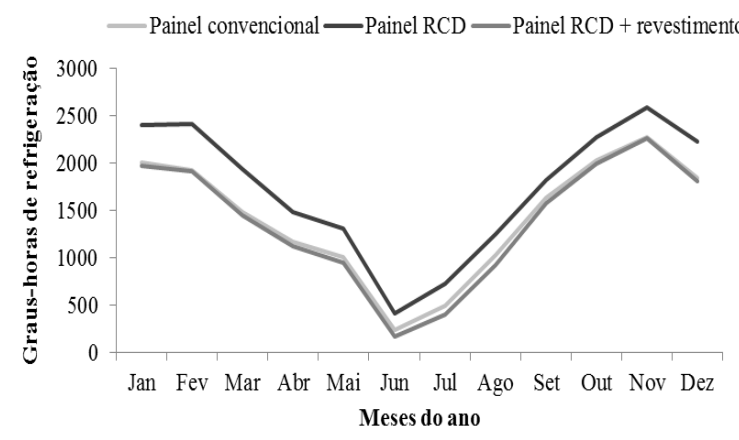

Figura 13 - Porcentagem de horas de desconforto anual dos paineis

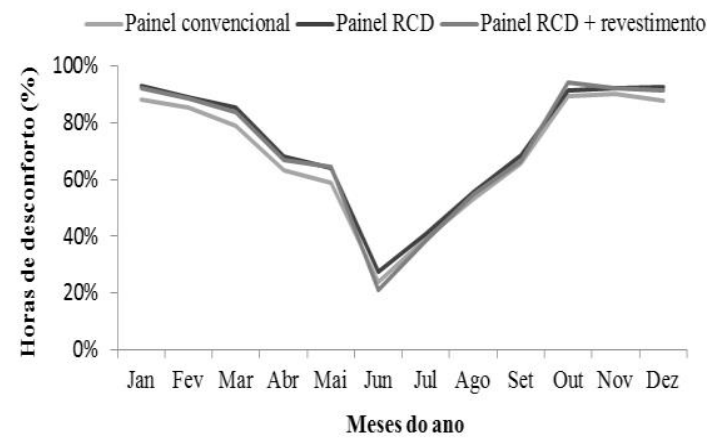

Nesse sentido, o uso de estratégias bioclimáticas é importante para melhorar a habitabilidade das edificações. Além destas, a melhoria do desempenho termoenergético e do conforto térmico pode ser alcançado a partir da adequação do isolamento térmico do envelope da edificação às condições climáticas da região. Isso significa adotar componentes que apresentem menores valores de transmitância térmica nos painéis de vedação e cobertura da edificação. Alterações nestes componentes implicam necessariamente na elevação de custos de implatação das HIS e requerem estudo específico, pois é um fator decisivo na implantação destas edificações. De qualquer forma, este custo deve ser incorporado no custo total visto que o uso de painéis convencionais (usuais) não tem garantido adequado nível de conforto térmico aos ocupantes destas edificações.

\section{CONCLUSÕES}

O ensaio na câmara térmica caracterizou a transmitância térmica do painel composto por blocos de $\mathrm{RCD}$ em $2,89 \mathrm{~W} / \mathrm{m}^{2} \mathrm{~K}$ e do painel de $\mathrm{RCD}$ revestido interna e externamente, em $2,5 \mathrm{~W} / \mathrm{m}^{2} \mathrm{~K}$. No ensaio da parede de bloco cerâmico revestido interna e externamente $(2,5 \mathrm{~cm})$ obteve-se valor de transmitância térmica de $2,40 \mathrm{~W} / \mathrm{m}^{2} \mathrm{~K}$, valor próximo ao teórico estabelecido pela NBR 15.220-3 (ABNT, 2003).

O desempenho termo energético da habitação de interesse social composta pelo painel RCD apresentou demanda anual de graus-horas de refrigeração $21,50 \%$ superior ao do painel convencional. Com intuito de reduzir essa demanda, foi agregado revestimento interno e externo ao bloco de RCD, reduzindo a demanda por refrigeração de 3,5\% em relação ao painel convencional. A classificação termo energética das habitações em painel convencional e RCD com revestimento externo e interno é Nível B, e do painel RCD sem revestimento é Nível C, de acordo com o RTQ-R.

Verificou-se que tanto a edificação com paredes tradicionais quanto as com blocos de RCD não garantiram adequado conforto térmico aos usuários, pois foram quantificadas em todos os meses, com exceção do mês de julho, $80 \%$ ou mais das horas dentro da faixa de desconforto. Assim, sugere-se que estratégias bioclimáticas sejam incorporadas ao projeto da HIS para melhorar sua habitabilidade e que sejam utilizados materiais com menor transmitância térmica dos fechamentos verticais e da cobertura da edificação, para melhor adequação às condições climáticas da região, para o que sugerese a utilização de novos materiais na composição do bloco de RCD. 


\section{AGRADECIMENTOS}

Os autores agradecem a Fundação Amparo à Pesquisa do Estado de Mato Grosso (FAPEMAT) (Processo $N^{\circ} 249353 / 2014$ ) pelo apoio financeiro a esta pesquisa.

\section{REFERÊNCIAS}

ASSOCIAÇÃO BRASILEIRA DE NORMAS TÉCNICAS (ABNT). NBR 15220: Desempenho Térmico de Edificações - Parte 3: Zoneamento bioclimático brasileiro e diretrizes construtivas para habitações unifamiliares de interesse social. Rio de Janeiro, 2003a.

ASSOCIAÇÃO BRASILEIRA DE NORMAS TÉCNICAS (ABNT). NBR 15220: Desempenho Térmico de Edificações - Parte 2: Métodos de cálculo da transmitância térmica, da capacidade térmica, do atraso térmico e do fator solar de elementos e componentes de edificações. Rio de Janeiro, 2003b.

ASSOCIAÇÃO BRASILEIRA DE NORMAS TÉCNICAS (ABNT). NBR 6136: Blocos vazados de concreto simples para alvenaria - Requisitos. Rio de Janeiro, 2004.

ASSOCIAÇÃO BRASILEIRA DE NORMAS TÉCNICAS (ABNT). NBR 15116: Agregados reciclados de resíduos sólidos da construção civil - Utilização em pavimentação e preparo de concreto sem função estrutural - Requisitos. Rio de Janeiro, 2004.

ASSOCIAÇÃO BRASILEIRA DE NORMAS TÉCNICAS (ABNT). NBR ISO 17025: Requisitos gerais para competência de laboratórios de ensaio e calibração. Rio de Janeiro, 2005.

BAKER, P. Historic Scotland Technical Paper 10: $U_{\mathbf{n}}$ values and traditional buildings. In situ measurements and their comparisons to calculated values. Glasgow Caledonian University. 2011.

BUYUKALACA, O.; BULUT, H.; YILMAZ, T. Analysis of variable-base heating and cooling degree-day for Turkey. Applied Energy, v. 68, n. 3, p. 269-283, 2001.

CALLEJAS, I. J. A.; DURANTE, L. C.; SILVA. R. A. F.; OLIVEIRA, A. S. Verificação dos requisitos normativos de desempenho térmico em habitação de interesse social implantada em zona bioclimática 7. In: Seminário Mato-grossense de Habitação de Interesse Social, 5, 2013, Cuiabá, Mato Grosso. Anais. Cuiabá, 2013.

COSTA, J. S. Agregados alternativos para argamassa e concreto produzidos a partir da reciclagem de rejeitos virgens da indústria de cerâmica tradicional. 2006. Tese de doutorado (Ciência e Engenharia de Materiais). UFSC. São Carlos.

GIVONI, B. Comfort, climate analysis and building design guidelines. Energy and Building, vol.18, 1992.

International Organization for Standardization (ISO). ISO 9869: Thermal insulation - Building elements - In-situ measurement of thermal resistance and thermal transmittance. 1994.

LEITE, F. C.; MOTTA, R. S.; VASCONCELOS, K. L., BERNUCCI, L. Laboratory evaluation of recycled construction and demolition waste for pavements. Construction and Building Materials, v. 25, p.2972-2979, 2011.

LEIVA, C.; SOLÍS-GUZMÁN. J.; MARRERO, M.; ARENAS, C. G. Recycled blocks with improved sound and fire insulation containing construction and demolition waste. Waste Management, v.33, p.663-671, 2013.

POON, C.S.; KOU, S. C.; LAM, L. Use of recycled aggregates in molded concrete bricks and blocks. Construction and Building Materials, v.16, v.281-289, 2002.

SOUTSOS, M. N.; TANG. K.; MILLARD. S. G. Concrete building blocks made with recycled demolition aggregate. Construction and Building Materials, v.25, p.726-735, 2011.

REGULAMENTO TÉCNICO DA QUALIDADE - RTQ-R. Nível de Eficiência Energética de Edificações Residenciais. 2010. Disponível: www.procelinfo.com.br/etiquetagem_edificios em: Acesso em 09/04/2014.

RUPP, R. F. Análise da transferência de calor em paredes compostas por tijolos maciços. 2008. Trabalho de Conclusão de Curso (TCC). Universidade Federal do Rio de Janeiro. 